\title{
Umbilical cord ulceration in association with intestinal atresia in a child with deletion 13q and Hirschsprung's disease
}

\author{
T Y Khong, W D A Ford, E A Haan
}

\begin{abstract}
The case is reported of a baby boy with an interstitial deletion of the long arm of chromosome 13 who, in addition to the described associations of Hirschsprung's disease and intestinal atresia, had umbilical cord ulceration resulting in massive intrapartum haemorrhage. This case provides support for the existence of a previously reported association between umbilical cord ulceration and intestinal atresia, and suggests that it is aetiologically heterogeneous.

(Arch Dis Child 1994; 71: F212-F213)
\end{abstract}

Three cases of congenital intestinal atresia, associated with linear ulcerations of the umbilical cord resulting in massive fetal haemorrhage, were recently reported. ${ }^{1}$ It was suggested that this may represent a newly recognised association. We report a further instance in a child with interstitial deletion of the long arm of chromosome 13 in whom additional abnormalities were found.

Department of Pathology, Women's and Children's Hospital, Adelaide T Y Khong

Department of Paediatric Surgery W D A Ford

Department of Medical Genetics and Epidemiology

E A Haan

Correspondence to Dr T Y Khong,

Department of Pathology,

Queen Victoria Hospital,

160 Fullarton Road,

Rose Park, Australia 5067.

Accepted 21 July 1994 rupture of membranes was performed and heavily bloodstained liquor was obtained. The fetal heart rate returned to $170-180$ beats per minute after this procedure. Normal vaginal delivery followed soon after, with the birth of a pale, male infant, weighing $1.63 \mathrm{~kg}$ with Apgar scores of 0,0 , and 0 at 0,5 , and 10 minutes. The baby was ventilated and, following cardiopulmonary resuscitation, a heart rate of 100-120 beats per minute was established at 20 minutes. A Kleihauer test was negative.

The placenta was of normal configuration and weight with no evidence of retroplacental haematoma. The amniochorial membranes were thickened up to about $15 \mathrm{~mm}$ by gelatinous oedematous fluid. Both umbilical arteries coursed extremely close to the surface of the cord and there were skip ulcerations in one of these arteries (fig 1). Histologically, both umbilical arteries showed thinning of the media on one side of the vessel, with abnormal medial cushioning in areas. One of the umbilical arteries was ulcerated with haemorrhage into perivascular spaces, Wharton's jelly, and, presumably, into the amniotic cavity (fig 2). There was no other pathology of the cord and placenta. It is presumed that the neonate was acutely anaemic due to a massive haemorrhage from ulceration of the umbilical artery in the cord.

Duodenal atresia was confirmed by abdominal radiography. At laparotomy on day 2 , five intestinal atresias were identified - in the second part of the duodenum resulting in a diverticulum, another in the second part of the duodenum, another at the junction of the distal duodenum and proximal jejunum, and two further areas in the proximal jejunum. The diverticulum and atretic areas were resected and a gastro-jejunostomy performed. The bile and pancreatic ducts drained into the proximal duodenum. The distal colon was noted to be small with inspissated meconium and there was polysplenia with three spleniculi. Subsequently, a gastrograffin enema failed to clear the meconium. A rectal biopsy was postponed because of difficulties in assessing ganglion cells in the immature infant. ${ }^{2}$

Feeding was gradually introduced but was never adequate, and on day 26 there was feeding intolerance, abdominal distension, and an abdominal mass. There was gradual recovery with intravenous antibiotics, cessation of feeding, and removal of the transanastomotic tube. Hirschsprung's disease was diagnosed subsequently on a rectal biopsy specimen performed on day 48 (corresponding to 38-39 weeks' gestation). A further contrast study was not required. At laparotomy,

Figure 1 Linear ulcerations with adherent clot along segment of umbilical cord (arrows). The cut end has been everted to show the superficially located vessels. 
Figure 2 Extravasated blood and blood clot overlying superficially located umbilical artery, showing thinning of one side.

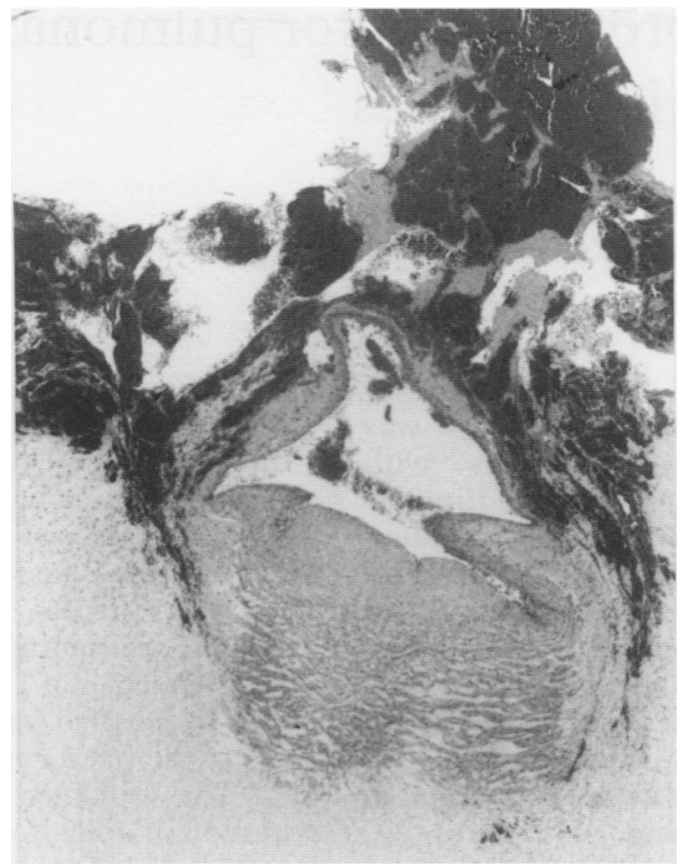

frozen sections confirmed that there was aganglionic bowel to the caecum and an ileostomy was performed in the ganglionic terminal ileum.

Computed tomography scans of the brain showed bilateral cerebral atrophy associated with deep white matter disease, consistent with perinatal asphyxia but with no evidence of ventriculomegaly. Echocardiography was normal.

Cytogenetic studies on the proband's blood showed an interstitial deletion of the long arm of chromosome 13: 46, XY, del (13)(q21.2q22). Parental chromosomes were normal. The child's facies were reviewed following the cytogenetic diagnosis; the only definite dysmorphic features were a narrow forehead, slightly upslanting palpebral fissures, and mild micrognathia. Ophthalmological review documented bilateral optic nerve hypoplasia, a recognised association of deletion 13q.

The child had recurrent bouts of aspiration pneumonia and died at the age of 10 months. Consent for necropsy was declined.

\section{Discussion}

This case provides further support for an association between intestinal atresia and ulceration of umbilical cord, as proposed by Bendon et al. ${ }^{1}$ These authors advanced three possible explanations for the association: vasospasm in both the umbilical artery and the vasculature supplying the gut, leading to ischaemic damage to both the vessel and gut walls; chemical ulceration of the umbilical cord secondary to reflux of gastric and duodenal contents as a result of upper gut atresia; and developmental abnormalities of the epithelial lining of both the gut and umbilical cord. Our case does not provide sufficient information to differentiate between these possibilities.

One of the three cases reported by Bendon and colleagues had congenital heart disease in addition to multiple jejunal atresias. Our patient's heart was normal clinically and on the echocardiogram reading. On the other hand, our infant had additional abnormalities, some of which were recognised associations of deletion 13q. The one patient karyotyped by Bendon et al had normal chromosomes. ${ }^{1}$

There are now several reports linking deletions from chromosome $13 \mathrm{q}$ with Hirschsprung's disease, with Hirschsprung's disease in the presence of jejunal atresia and duodenal atresia, and with agenesis of the ascending colon. ${ }^{3-6}$ The site of deletion of chromosome $13 \mathrm{q}$ in our proband overlaps with others described: q14.1q22.3, ${ }^{3}$ q21.2q32.3, $\mathrm{q} 22.1 \mathrm{q} 32.1,{ }^{4}$ and q22qter. ${ }^{5}$ Another $13 \mathrm{q}$ deletion associated with Hirschsprung's disease is q32.3q33.2.6 Our patient's chromosomal abnormality was probably causally related to the Hirschsprung's disease, intestinal atresias, and the umbilical cord ulceration. The pathogenetic sequence and mechanism(s) are unknown but may be shared with those cases of intestinal atresia and cord ulceration that are chromosomally normal.

Bendon and colleagues ${ }^{1}$ suggested that the association may be more common than is thought. We believe, however, that the association is rare. In our 15 years of experience examining placentas in hospitals with between 3000 and 5000 deliveries a year, ulceration of the umbilical cord has never been found. Another chromosome abnormality associated with intestinal atresia (and Hirschsprung's disease) is trisomy 21 but, to our knowledge, umbilical cord ulceration has not been described in this context. Furthermore, neonates born with intestinal atresia usually do not have evidence of antepartum or intrapartum bleeding. Further cases of the association will allow the range of situations in which it can occur and its pathogenesis to be documented.

\section{Addendum}

Two cases of isolated umbilical cord ulceration thought to be due to cord compression and meconium staining were overlooked in our original review of the published data: Altshuler $G$, Arizawa M, Molnar-Nadesdy G. Meconiuminduced umbilical cord vascular necrosis and ulceration: A potential link between the placenta and poor outcome. Obstet Gynecol 1992: 79: 760-6.

We thank Mr Ross Sweet and Dr R Vigneswaran who provided consultant obstetric and neonatal care, respectively, for permission to report this case.

1 Bendon RW, Tyson RW, Baldwin VJ, Cashner KA, Mimouni F, Miodovnik M. Umbilical cord ulceration and intestinal atresia: A new association? Am $\mathcal{F}$ Obstet Gynecol 1991; 164: 582-6.

2 Smith B. Pre- and postnatal development of the ganglion cells of the rectum and its surgical implications. F Pediatr Surg 1968; 3: 386-91.

3 Sparkes RS, Sparkes MC, Kalina RE, Pagon RA, Salk DJ, Disteche CM. Separation of retinoblastoma and esterase $\mathrm{D}$ loci in a patient with sporadic retinoblastoma and del (13)(q14.1q22.3). Hum Genet 1984; 68: 258-91.

4 Lamont MA, Fitchett M, Dennis NR. Intestinal deletion of distal $13 \mathrm{q}$ associated with Hirschsprung's disease. $\mathcal{F}$ Med Genet 1989; 26: 100-4.

5 Kiss P, Osztovics $M$. Association of $13 q$ deletion and Hirschsprung's disease. $\mathcal{F}$ Med Genet 1989; 26: 793-6. 6 Bottani A, Xie Y, Binkert F, Schinzel A. A case microdeletion, del (13)(q32.3q33.2): potential mapping of one disease locus. Hum Genet 1991; 87: 748-50. 